\title{
Easy Pulsatile Phantom for Teaching and Validation of Flow Measurements in Ultrasound
}

Authors

Affiliations

\section{B. Rominger', E.-M. Müller-Stuler², M. Pinto², A. S. Becker², K. Martini², T. Frauenfelder², V. Klingmüller}

${ }^{1}$ Institute of Diagnostic and Interventional Radiology, UniversitätsSpital Zurich, Zürich, Switzerland

${ }^{2}$ Institute of Diagnostic and Interventional Radiology, University Hospital Zurich, University of Zurich, Zurich, Switzerland
Key words

education

ultrasound

- blood vessels

- ultrasound spectral doppler

- ultrasound color doppler received $\quad 10.08 .2015$

accepted 28.03.2016

Bibliography

DOI http://dx.doi.org/

10.1055/s-0042-106396

Published online:

July 13, 2016

Ultrasound International Open

2016; 2: E93-E97

(c) Georg Thieme Verlag KG

Stuttgart · New York

ISSN 2199-7152

\section{Correspondence}

Prof. Marga B Rominger, MD

Institute of Diagnostic and Interventional Radiology

UniversitätsSpital Zurich

Rämistrasse 100

8006 Zürich

Switzerland

Tel.: + 41/442/552 900

Marga.Rominger@usz.ch

\section{Abstract \\ $\nabla$}

Purpose: To build a simple model to teach and validate non-pulsatile and pulsatile flow quantification in ultrasound.

Materials and Methods: The setting consists of the following connected components: (1) medical syringe pump producing an adjustable constant flow ( $\mathrm{ml} / \mathrm{min}),(2)$ modulator modifying constant flow to a reproducible pulsatile flow, (3) water tank containing a diagonal running silicone tube $(0.5 \mathrm{~mm}$ inner diameter $)$, and (4) a fixated ultrasound probe (L9 Linear Array $9 \mathrm{MHz}$, GE Logiq E9) measuring the flow inside the tube. Commercially available microbubbles suspended with physiological saline solution were used for ultrasonic visibility. Spectral Doppler of different flow profiles is performed.

\section{Introduction}

$\nabla$

Ultrasound is widely used in medicine by many disciplines for the qualitative and quantitative evaluation of vessels and parenchyma perfusion. There are many operator-dependent technical parameters that need to be known to improve the accuracy of flow and perfusion measurements. Skills labs for medical students and hands-on training of doctors are becoming more and more popular. Yet, there is a lack of compact models to teach and validate flow measurement in ultrasound.

There are a variety of ultrasound measurements that allow the assessment of velocities within a vessel. The spectral analysis of the Doppler ultrasound signal through Fast Fourier Transformation delivers the velocities within the vessel, which can be used to calculate the mean velocity per cycle.

Medical syringe pumps are standard equipment in the medical field and generate a highly accu-
Results: The syringe pump produces an adjustable, constant flow and serves as the reference standard. The filling volume of the tube system is $1.2 \mathrm{ml}$. Microbubbles are very well detected by ultrasound and can be used as an easy and clean blood mimicking substance. The modulator generates different physiological and pathological flow profiles. Velocities are similar to those found within human blood vessels. Thus, it is possible to train and validate flow measurements in ultrasound.

Conclusion: The model produces non-pulsatile and various pulsatile flow profiles and allows validation of flow measurements. The compact size permits easy and economic setup for flow measurements in research, skills lab and continuing education. 


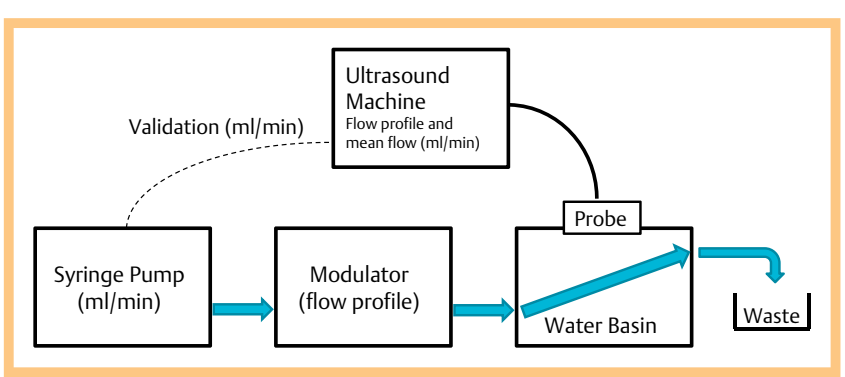

Fig. 1 Block diagram of pulsatile flow phantom.

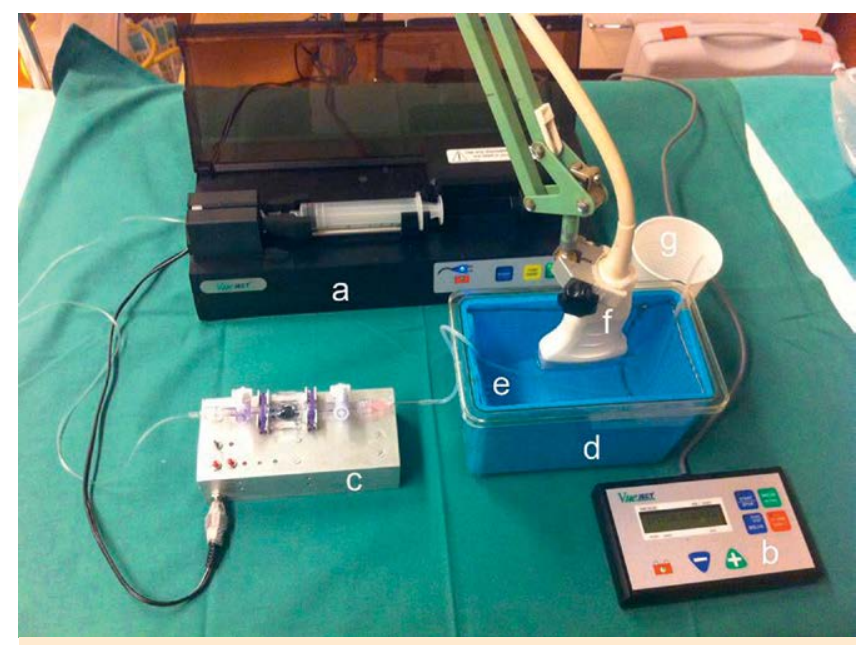

Fig. 2 Setup of pulsatile flow phantom: a medical syringe pump, $\mathbf{b}$ console terminal, $\mathbf{c}$ modulator, $\mathbf{d}$ water tank, e silicone tube, $\mathbf{f}$ probe, and $\mathbf{g}$ collecting tray.

Table 1 Components of pulsatile flow phantom.

\section{Components of pulsatile flow phantom}

Medical syringe pump (Vueject ${ }^{\mathrm{TM}}$, Bracco Suisse SA)

Connecting tube (Vueject Kit, Bracco Suisse SA)

Modulator (self-made component; see appendix for construction details)

Silicone tube (Keller Elastomere, Überlingen, Germany)1)

Peripheral venous catheter for connection of silicone tube with modulator (BD Venflon $\left.{ }^{\mathrm{TM}}, 20 \mathrm{G}\right)$

Microbubbles (SonoVue ${ }^{\mathrm{TM}}$, Bracco Suisse SA) ${ }^{2)}$

Water bin (household ware, filled with tap water)

Wire frame (formed from wire for sponge support and tube guidance)

Thin sponges (household ware, liners for water bin inside wall to prevent ultrasound reflection)

Rack for ultrasound probe (desk lamp arm with a clamp)

Collecting tray

For acoustic and mechanical properties of vessel- and blood-mimicking substances, refer to the literature ${ }^{1)}[2],{ }^{2)}[15,17]$

running at a constant flow. Microbubbles suspended in physiological saline solution guaranteed the ultrasonic visibility of the flow within the tube. The flow in the silicone tube was measured by a linear probe that was inserted into the water basin ( $\bullet$ Fig. 1,2, $\odot$ Table 1 ).

\section{Materials}

A dedicated syringe pump for microbubbles (VueJECT ${ }^{\mathrm{TM}}$, Bracco Suisse SA) was used to produce a constant flow. The flow ranged from $0.3 \mathrm{ml} / \mathrm{min}$ to $4.0 \mathrm{ml} / \mathrm{min}$ in $0.1 \mathrm{ml} / \mathrm{min}$ increments on a
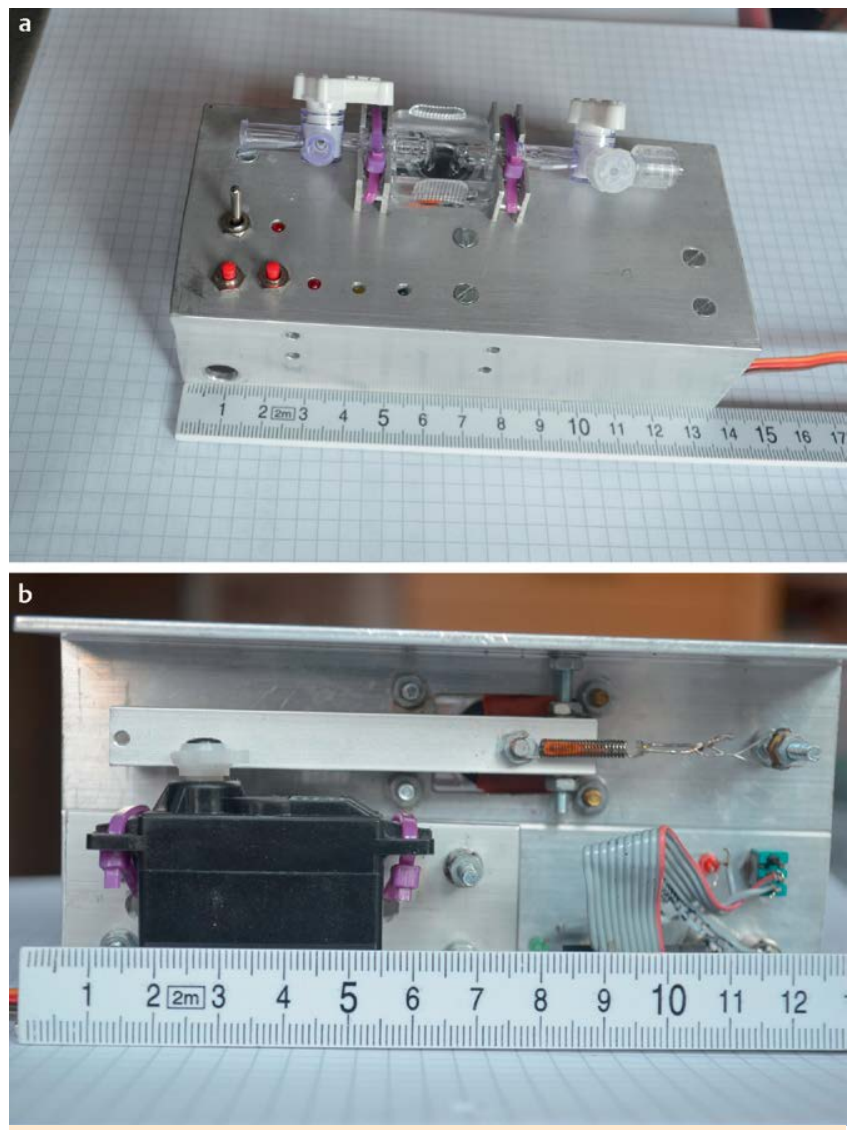

Fig. 3 Modulator: View from the $\mathbf{a}$ top and $\mathbf{b}$ bottom.

console terminal. For ultrasound visibility, SonoVue ${ }^{\mathrm{TM}}$ (Bracco Suisse SA) was freshly prepared according to the information and instructions of the manufacturer. $1 \mathrm{ml}$ from this $5 \mathrm{ml}$ of SonoVue ${ }^{\mathrm{TM}}$ solution was further diluted with $0.9 \%$ physiological saline fluid (1:200). A $20 \mathrm{ml}$ Luer Lock syringe (B. Braun OriginalPerfusor $^{\circledR}$ OPS) served as the reservoir. The size of the syringe pump measured $33.7 \times 14.5 \times 10.1 \mathrm{~cm}$, and the size of the console was $14.5 \times 8.5 \times 2.3 \mathrm{~cm}$. The weight of the syringe pump and console together was $3.9 \mathrm{~kg}$. The accuracy of the syringe pump was specified with $\pm 2 \%$ (Handbook; VueJECT ${ }^{\mathrm{TM}}$, Bracco Suisse SA). Downstream a self-made modulator (N.N.) transformed the non-pulsatile flow into a pulsatile flow ( $\bullet$ Fig. 3). The modulator consisted of a motor (Servo Motor DS 8077 BB, Graupner, Germany) that drove a lever arm. This compressed a reservoir which is normally used for arterial blood pressure measurements in the intensive care unit [1]. The maximum volume of compression was $24 \mu \mathrm{l}$ per pulse. The servo motor was controlled by the microcontroller ATmega8-16PI. The modulator measured $13.5 \times 6.5 \times 3.5 \mathrm{~cm}$ with a weight of $600 \mathrm{~g}$. Further details of the modulator including wiring and construction plan as well as software are available via email. The basic cycle time of the lever arm was $1 \mathrm{~Hz}$ ( = pulse 60) and can be modified between 40 and 120 beats/min. The basic software version allowed the storing of 6 different flow profiles in the microcontroller. Other profiles can be generated on a personal computer and transferred to the microcontroller via the serial interface RS232.

We placed the test tube in a plastic water basin (volume: 1.31) for ultrasound imaging. The silicone tube that was used for ultrasound flow imaging had an inner lumen diameter of 0.5 $\mathrm{mm}$ (area of inner lumen $=0.00196 \mathrm{~cm}^{2}$ ) and a wall thickness of 


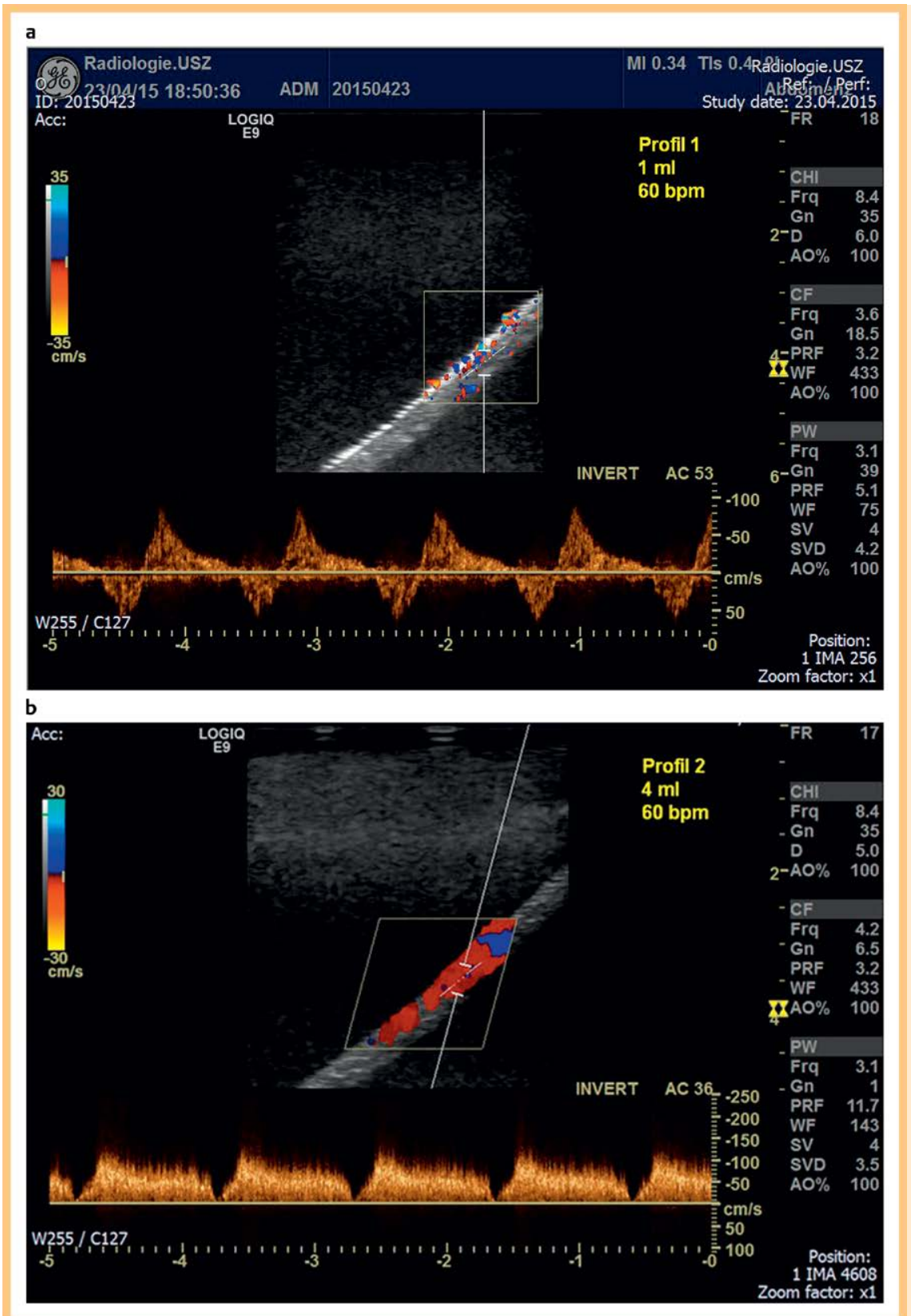

Fig. 4 Triplex of 2 different flow profiles a with $1 \mathrm{ml} / \mathrm{min}$ flow and $\mathbf{b}$ with $4 \mathrm{ml} / \mathrm{min}$; both 60 beats per minute.

$1 \mathrm{~mm}$ (Keller Elastomere, Überlingen, Germany). The internal walls of the basin were covered with thin household sponges to prevent the reflection of the ultrasound signals. A wire construction secured the sponges to the basin walls and held the tube diagonally through the basin with a constant angle of $60^{\circ}$. The basin was filled with tap water. To minimize air bubbles, the water was cooked and after it cooled was poured gently into the basin.

\section{Ultrasound imaging and analysis}

We used the GE Logiq E9 ultrasound system with a $9 \mathrm{MHz}$ linear probe L9 (General Electric Healthcare, Switzerland) for image and data acquisition. The ultrasound probe was attached to a flexible arm, fixed to an infusion stand. Color and spectral Doppler of the different profiles with varying flow was acquired after $3 \mathrm{~s}$ for equilibrium flow.

\section{Results and Discussion}

$\nabla$

The pulsatile flow model allowed an easy and economic setup for flow teaching and validation ( $\bullet$ Fig. 1, 2). A medical syringe pump was used for adjustable and exact flow generation. The downstream, independent flow modulator formed various pulsatile flow profiles ( $\odot$ Fig. 4). The modulator accelerated the flow velocity by compression of a reservoir with a lever arm or retarded the velocity by depression of the same reservoir. Faster compression of the reservoir produced a higher peak velocity, while fast depression produced a back flow. Changing the constant flow of the syringe pump led to a modification of the flow profile. Hence, the flow profile induced by the lever arm of the downstream modulator is dependent on the gross volume of the syringe pump. The syringe pump and downstream modulator can be regulated independently. High velocities were achieved by combining the syringe pump and flow modulator with very thin tubes. The constant flow $(\mathrm{ml} / \mathrm{min})$ of the syringe pump 
Table 2 Validation of results of spectral Doppler analysis.

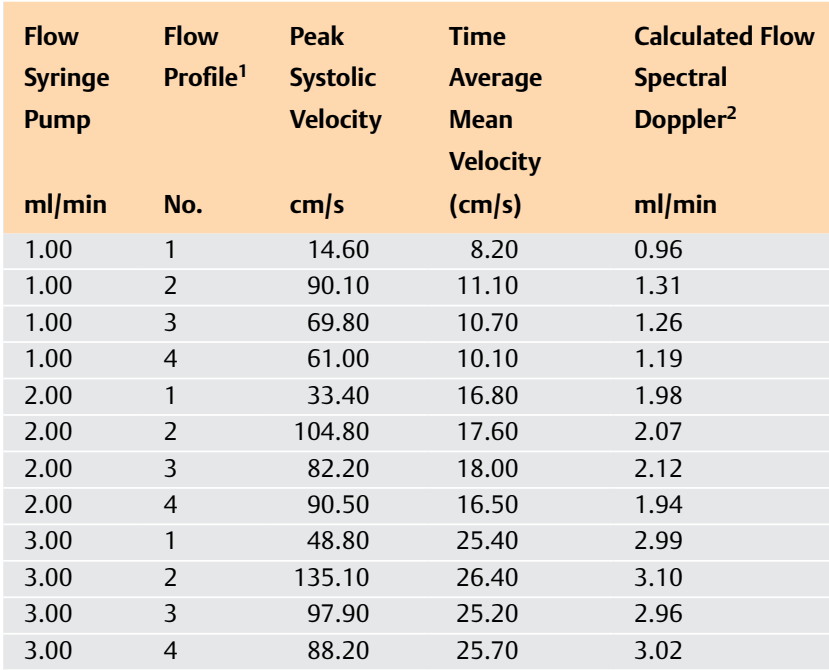

${ }^{1}$ Flow profile 1 was constant flow and flow profiles $2-4$ were different pulsatile flow profiles formed by the modulator. ${ }^{2}$ The flow in the last column is calculated from the time average mean velocity and the known area of the inner lumen of the tube. Thus the flow from spectral Doppler can be compared to the flow of the syringe pump in column one

served as an internal reference for the goodness of mean flow ultrasound measurements as well as manufacturer and external software analysis programs ( $\bullet$ Table 2 ). We performed repeated measurements over $5 \mathrm{~min}(60 \mathrm{bpm}, 1 \mathrm{~Hz})$ for one pulsatile flow profile at a medical syringe pump rate of $1 \mathrm{ml} / \mathrm{min}$. The mean velocity was recorded every $10 \mathrm{~s}$ by the ultrasound machine. The calculated average flow was $0.97 \mathrm{ml} / \mathrm{min}$ (standard deviation 0.029). Müller-Stüler [1] showed constancy of mean flow and velocities for repeated measurements using a syringe pump and modulator.

\section{Syringe pump}

Syringe pumps have been widely used in medicine to generate variable adjustable flow and exact constant flow. Medical syringe pumps are highly accurate, very reliable, as well as durable equipment. Our syringe pump was a dedicated medical syringe pump for the infusion of microbubbles. A standard medical syringe pump in combination with a vibrating table to maintain fluid-mimicking substances in suspension, as used for example in laboratories, can also be used [1]. Syringe pumps that are not in clinical use might still be exact enough for teaching.

\section{Modulator}

To transform a constant flow into a pulsatile flow, we used a simple, self-made modulator. This consisted of a motor-driven lever arm compressing a reservoir. The hardware of the modulator is an open system. It is attached to a workbench allowing easy access to all built-in components ( $\bullet$ Fig. 3 ). The modulator produces different physiological and pathological flow profiles. 3 cycles were needed to get a constant equilibrium of flow profiles. Complex flow profiles including ante- and retrograde flow and maximum peak velocities within the range of human vessels $(135 \mathrm{~cm} / \mathrm{s}$ peak velocity for $3 \mathrm{ml} / \mathrm{min}$ flow) could be achieved (O Fig. 4).

The open access to the modulator on a workbench allowed easy exchange of components. This is a major advantage over closed systems that may be required to be sent in for repair. The total cost of all modulator components was about $100 €$.
Tubes and water tank

We used commercially available very thin silicon tubes, which we tested beforehand for ultrasound transmissibility. The filling volume of the complete tube system without the syringe was $1.2 \mathrm{ml}$. For improved transmissibility, we recommend the use of polyvinyl chloride (PVC) tubes [2-4]. Around the inner walls of the water tank, we prefer thin sponges to small stones at the bottom [5] to minimize ultrasound reflection. Several tissue-mimicking substances to surround the tube in the tank can be used $[1,2,4,6-8]$. Yet, we found tap water gently poured into the basin to be easiest, cheapest and sufficient for our measurements. Instead of tap water a $9 \%$ glycerol solution can be chosen to mimic the sound velocity of soft tissue [2]. We changed the water daily. For longer use we recommend adding an antiseptic substance, for example Microsept ${ }^{\circledR}$. The small syringe reservoir together with thin tubes allowed ultrasound measurements not disturbed by large air bubbles. In the case of an air bubble adherent to the outer tube wall within the measurement field, we gently removed it from the tube with a small hooked instrument (for example, a bent paper clip).

\section{Blood-mimicking fluids}

Several blood-mimicking fluids can be used for the ultrasonic visibility of tube content $[1,2,4,9-12]$. We preferred microbubbles, which are commonly used for contrast-enhanced examinations in an ultrasound department $[13,14]$. Microbubbles are within the range of size of red blood cells and are known to facilitate Doppler ultrasound in human application [14]. Tortoli et al. [15] investigated the use of microbubbles in Doppler ultrasound. They found that the microbubble phenomena due to radiation force produce evident Doppler effects in vitro, but do not interfere with Doppler examination. For teaching purposes, expired or leftover microbubbles can be used. Microbubbles were gently handled by the dedicated syringe pump and the reservoir of the modulator. The small syringe reservoir and thin tube size allowed economic use of microbubbles without disturbing air bubbles within the tube system. We found a high concentration of microbubbles too noisy for spectral Doppler analysis (spectral broadening). To a certain degree gain reduction can reduce spectral broadening [16]. In the case of not freshly prepared microbubbles (e.g., leftover vials from human application), less dilution is necessary.

Alternatively, ultrasound blood-mimicking fluids (e.g., BMF-US, Shelley Medical Imaging Technologies, Ontario, Canada) can be ordered. The latter contains nylon particles with a size of $5 \mu \mathrm{m}$ and has the rheological characteristics of blood. These particles were less echoic than microbubbles. Due to the small volume needed by this flow phantom model, freshly drawn blood can be used [1].

\section{Limitations \\ $\nabla$}

For teaching and validation, we found it sufficient to use tap water with low gas content for surrounding tissue mimicking, silicone tubes for vessel mimicking, as well as microbubbles for blood mimicking. Yet, for research purposes replacement by more similar tissue-, vessel-, as well as blood-mimicking substances with respect to physical, acoustic, and rheological properties on spectral Doppler analysis has to be considered. The precise mean flow of the syringe pump can be used for validation of the mean flow measured by ultrasound. The systolic peak 
and diastolic velocity of a pulsatile flow profile cannot be directly validated. The potential of our easy phantom model for Doppler quality assurance has to be assessed by further studies. Our flow model is a multimodality pulsatile flow phantom and can be used for computed tomography and angiography as well. However, due to some magnetic components, it is not yet MRI-compatible.

In conclusion, our phantom generates non-pulsatile and various pulsatile flow profiles and served as an internal reference for mean flow ultrasound measurements. The handy size allowed clear and economic setup for flow quantification experiments, skills lab and hands-on teaching, as well as for standardized examinations.

\section{References}

1 Müller-Stüler E. Mathematical Analysis of Doppler signal for quantitative assessment of blood flow Mathematische Analyse des Dopplersignals zur quantitativen Bestimmung des Blutflusses. Diss. Aachen: Shaker Verlag; 2012

2 Hoskins PR. Simulation and validation of arterial ultrasound imaging and blood flow. Ultrasound in medicine \& biology 2008; 34: 693-717

3 King DM, Moran CM, McNamara JD et al. Development of a vesselmimicking material for use in anatomically realistic Doppler flow phantoms. Ultrasound in medicine \& biology 2011; 37: 813-826

4 Browne JE. A review of Doppler ultrasound quality assurance protocols and test devices. Phys Med 2014; 30: 742-751

5 Menke $M$. [Flow assessment trough contineous intensity measurement of back-scatter echos in a phantom modell] Volumenflussbestimmung durch kontinuierliche Intensitätsmessung der Rückstreuechos in einem Flussphantom. Diss. Charité-Universitätsmedizin Berlin 2012 http://www.diss.fu-berlin.de/diss/servlets/MCRFileNodeServlet/FUDISS_derivate_000000011412 /Dissertation_Mareile_Menke_2. Onlineversion.pdf

6 Browne JE, Ramnarine KV, Watson AJ et al. Assessment of the acoustic properties of common tissue-mimicking test phantoms. Ultrasound in medicine \& biology 2003; 29: 1053-1060
7 Dong F, Madsen EL, MacDonald MC et al. Nonlinearity parameter for tissue-mimicking materials. Ultrasound in medicine \& biology 1999; 25: 831-838

8 Madsen EL, Frank GR, Dong F. Liquid or solid ultrasonically tissuemimicking materials with very low scatter. Ultrasound in medicine \& biology 1998; 24: 535-542

9 Uddin S. Multimodal phantom model for evaluation of small vessels Multimodales Phantommodell für die Evaluierung von Stenosen in kleinen Gefässen, Diss. University Giessen. 2003

10 Oates $C P$. Towards an ideal blood analogue for Doppler ultrasound phantoms. Physics in medicine and biology 1991; 36: 1433-1442

11 Ramnarine KV, Hoskins PR, Routh HF et al. Doppler backscatter properties of a blood-mimicking fluid for Doppler performance assessment. Ultrasound in medicine \& biology 1999; 25: 105-110

12 Ramnarine $K V$, Nassiri DK, Hoskins PR et al. Validation of a new bloodmimicking fluid for use in Doppler flow test objects. Ultrasound in medicine \& biology 1998; 24: 451-459

13 Claudon M, Dietrich CF, Choi BI et al. Guidelines and good clinical practice recommendations for contrast enhanced ultrasound (CEUS) in the liver - update 2012: a WFUMB-EFSUMB initiative in cooperation with representatives of AFSUMB, AIUM, ASUM, FLAUS and ICUS. Ultraschall in der Medizin (Stuttgart, Germany: 1980) 2013; 34: 11-29

14 Piscaglia F, Nolsoe C, Dietrich CF et al. The EFSUMB Guidelines and Recommendations on the Clinical Practice of Contrast Enhanced Ultrasound (CEUS): update 2011 on non-hepatic applications. Ultraschall in der Medizin (Stuttgart, Germany: 1980). 2012; 33: 33-59

15 Tortoli P, Guidi F, Mori $R$ et al. The use of microbubbles in Doppler ultrasound studies. Medical \& biological engineering \& computing 2009; 47: 827-838

16 Logallo N, Fromm A, Waje-Andreassen U et al. Effect of microbubble contrast on intracranial blood flow velocity assessed by transcranial Doppler. Journal of ultrasound 2014; 17: 21-26

17 Gorce JM, Arditi M, Schneider M. Influence of bubble size distribution on the echogenicity of ultrasound contrast agents: a study of SonoVue. Investigative radiology 2000; 35: 661-671 